\title{
Absorptionsmessungen an Lösungen von Neodymsalzen
}

\author{
Von G. W. Oetsen ${ }^{1}$
}

(Z. Naturforschg. 4 a, 1-10 [1949]; eingegangen am 22. April 1948)

\begin{abstract}
Absorptionsmessungen an 5 Neodymsalzen in Abhängigkeit von der Konzentration zeigen zwei verschiedene Effekte: a) Schwankungen der Absorptionskonstanten und Oszillatorenstärken um kleine Beträge bej Änderung der Konzentration. Sie werden auf Umbauvorgänge in den äußeren Schichten der Hydrathülle des Nd-Ions zurückgeführt. Der Effekt tritt bei allen Salzen auf. b) Eine starke Zunahme der Absorptionskonstanten und Oszillatorenstärken der Bande D mit wachsender Konzentration bei den Nitraten. Der Effekt tritt nicht beim Chlorid und Perchlorat, andeutungsweise beim Bromat auf. Er wird gedeutet als radikale Änderung der Struktur in der nächsten Umgebung des NdIons (Komplexbildung). Entscheidend für diesen Effekt scheint der sterische Aufbau des Anions zu sein. - Es werden weiter Dichtemessungen mitgeteilt. Die durch den Schwankungseffekt a) optisch ausgezeichneten Konzentrationen scheinen auch Anomalien der Dichtekurve zu lieferh. Hierdurch wird die Rückführung der optischen Befunde auf strukturelle Eigenschaften der Lösungen gestützt.
\end{abstract}

$\mathrm{S}$ chon mehrfach ist auf die Bedeutung hingeWwiesen worden. die die Ionen der Seltenen Erden als Sonden zur Erforschung des strukturellen Aufbaues fester und flüssiger Substanzen gewinnen können ${ }^{2}$. Die charakteristischen Spektren dieser Ionen müssen Übergängen innerha!h, der $4 f$-Schale zugeordnet werden, d. h. die beteiligten Terme unterscheiden sich nur in den Quantenzahlen $S, L$ und $J$, nicht jedoch in der Elektronenkonfiguration $4 f^{n}$ (wobei $n=1 \ldots 13$ von Ce... Yb). Beim Einbau eines Seltenen-Erd-Ions in einen Kristall oder in eine Lösung kommen die $4 f$-Elektronen unter den Einfluß eines von den Nachbarmolekeln und -ionen erzeugten elektrischen Störfeldes. Diese Störung bewirkt erstens eine durch die Feldsymmetrie bestimmte Aufspaltung der Linien und zweitens eine durch Symmetrie und Stärke des Feldes bestimmte Änderung der Linienintensität, vor allem bei der elektrischen Dipolstrahlung, die im freien Ion durch die Laporte-Regel verboten ist und erst durch das Störfeld der Umgebung erzwungen wird $^{3}$.

Jede strukturelle Anderung in der Umgebung eines der Seltenen-Erd-Ionen muß sich also verfolgen lassen, entweder an Änderungen in der Zahl und Lage der Aufspaltungskomponenten

1 D 7. Anschrift des Verf: Köln-Bayental, Bonner Straße.

${ }^{2}$ R. T o m a s c h e k, Ergebn. exakt. Naturwiss. 20, 286 [1942].

3 J. H. v a n V le ck, J. physic. Chem. 41, 167 [1937].

${ }^{4}$ K. H. H e 1 l w e g e, Ann. Physik 40, 530 [1941].

oder an der Intensität der elektrischen Dipolstrahlung. Jedoch lassen sich die einzelnen Komponenten bei Zimmertemperatur nur bei denjenigen Seltenen Erden getrennt beobachten, deren Linien wegen besonders geringer Kopplung der $4 f$-Elektronen an das Gitter ${ }^{4}$ extreme Schärfe haben, wie z. B. beim Europium-Ion. Bei den anderen Seltenen Erden, z. B. beim Neodym, sind bei Zimmertemperatur die Linien so breit, daß sie zu Banden verschmelzen. Hier muß die Intensität der elektrischen Dipolstrahlung als Kriterium herangezogen werden.

Die älteren Messungen an Lösungen von Salzen der Seltenen Erden sind vorwiegend zu chemischanalytischen $\mathrm{Z}$ wecken durchgeführt worden ${ }^{5}$. Sie benutzen rein qualitative, visuelle oder photographische Methoden, sind also, obwohl sie einzelne Hinweise liefern, im Rahmen der oben entwickelten theoretischen Vorstellungen unbrauchbar. Dagegen haben G o r t e r und Mitarbb. ${ }^{6}$ eine quantitative Ausmessung der Absorptionsspektren einiger Praseodym-, Neodym- und Samariumsalze zwischen $11000 \mathrm{~cm}^{-1}$ und $23000 \mathrm{~cm}^{-1}$ durchgeführt und die Oszillatorenstärken der verschiedenen Banden bei gewissen einzelnen Konzentrationen bestimmt. Bisher sind diese Messungen $\mathrm{m}$. W. jedoch nicht theoretisch ausgewertet worden.

5 z. B. Yntema, J. Amer. chem. Soc. 45/I, $90 \dot{7}$ [1923]; L. L. Quill, P. W. Selwood u. B. S. Ho p k in s, J. Amer. chem. Soc. 50/II, 2929 [1928].

${ }_{6}$ C. J. G or t e r u. Mitarbb., Physica 9, 217 [1942]; 10, 365 [1943]. 
Einen großen Fortschritt in dieser Richtung haben die Arbeiten von F r e e d und Mitarbb. ${ }^{7}$ an Lösungen von Europiumsalzen gebracht. Beobachtet wurde die Zahl der Aufspaltungskomponenten, was - wie oben gesagt - beim Europium auch in Lösungen möglich ist. Es zeigte sich, daß in Lösungen ein ebenso klares Aufspaltungsbild vorhanden ist wie in Kristallen, woraus geschlossen werden muß, daß die Umgebung der Europium-Ionen in Lösungen keineswegs statistisch diffus ist, sondern eine wohl definierte Symmetrie (Linienzahl) und Störfeldstärke (Größe der Aufspaltung) besitzt. Die so definierte Umgebung des Seltenen-Erd-Ions hängt ab vom Lösungsmittel und vom Anion. In wäßriger Lösung ist sie bei sehr starker Verdünnung vom Anion unabhängig. Bei Chloridlösungen ändert sich dann nach F re ed ${ }^{8}$ auch mit einer bis zur Sättigung wachsenden Konzentration die Symmetrie gar nicht, während beim Nitrat oberhalb von 0,01- $m$. die Umgebung einer mit der Konzentration wachsenden Zahl von Ionen eine neue, ganz • andere Struktur annimmt, bis in der Nähe der Sättigung alle Ionen die neue Struktur besitzen.

Dieser „Nitrat-Effekt" ist von Chow und $\mathrm{Hellwege} \mathrm{e}^{\mathbf{a}}$ auch an der Bande $\mathrm{D}^{10}$ (Wellenlänge $=5800 \AA$ ) des NdZn-Nitrats gefunden worden, da hier offenbar die beiden verschiedenen Umgebungen eine um den Faktor 2 verschiedene Oszillatorenstärke der elektrischen Dipolstrahlung erzwingen, was wegen des konzentrationsabhängigen Mischungsverhältnisses der beiden „Ionen-Formen“ eine sehr starke Abhängigkeit der beobachteten Oszillatorenstärke von der Konzentration zur Folge hat. Im Spektrum des Neodym-Ions in Lösung heben sich die einzelnen Linien nur noch als schwache Buckel aus den entstehenden Banden heraus, so daß die Änderung des Aufspaltungsbildes mit der Konzentration hier nicht verfolgt werden konnte.

Die vorliegende Untersuchung hat das Ziel, die Messung der Absorptionskonstanten und Oszil-

7 S. F r e ed u. Mitarbb., J. chem. Physics 6, 297, 654, 655 [1938]; 7, 824 [1939]; 8, 247, 291, 840, 878 [1940]; J. Amer. chem. Soc. 63, 1079 [1941].

${ }^{8}$ Es wurde nur auf die Zahl der Linien geachtet; Intensitäts- und Linienbreitenmessungen wurden jedoch nicht durchgeführt, so daß foinere Effekte evtl. der Beobachtung entgangen sind.

9. K. Ch ow u. K. H. H ell w e g e, Z. Physik 125, 18 [1948]. latorenstärke in Abhängigkeit von der Konzentration auf möglichst viele Neodymsalze mit verschiedenen Anionen auszudehnen, um die Frage zu beantworten, wieweit die Struktur in der Umgebung des Neodym-Ions durch das Lösungsmittel und wieweit sie auch durch die Anionen bestimmt wird. Außerdem sollten zwei verschiedene Absorptionsbanden untersucht werden, um von den Zufälligkeiten eines speziellen Úberganges freizukommen.

\section{M e $ß$ anordnung}

Da Vorversuche ergeben hatten, daß die Unterschiede der Oszillatorenstärken bei verschiedenen Anionen voraussichtlich in der Gegend von 10 bis $20 \%$ lagen, handelte es sich darum, eine genauere und schnellere Meßmethode anzuwenden als das von $\mathrm{Chow}$ und $\mathrm{Hellw}$ ege ${ }^{9}$ benutzte photographische Verfahren.

Mit Hilfe einer unten näher beschriebenen photoelektrischen Methode ist es gelungen, bei einer spektralen Spaltbreite von $2-4 \AA$ etwa 70 Meßpunkte über eine Bande (Halbwertsbreite $95 \AA$ ) zu verteilen. Dabei wurde an jeder Stelle das Verhältnis der bei der absorbierenden Schicht ein- und austretenden Strahlungsleistung $I_{0} / \boldsymbol{I}$ auf $\pm 3 \%$ genau gemessen ${ }^{11}$. Wegen der Schwierigkeit bei der Konzentrations- und Schichtdickenbestimmung waren die molare Absorptionskonstante

$$
\begin{aligned}
h \cdot(\tilde{v}) & =\frac{1}{c \cdot d} \ln \frac{I_{0}}{I}\left[\text { Liter } \cdot \mathrm{Mol}^{-1} \mathrm{~cm}^{-1}\right] \\
c & =\text { Konzentration }\left[\frac{\mathrm{Mol} \mathrm{Nd}^{+++}}{\mathrm{Liter}}\right] \\
d & =\text { Schichtdicke }[\mathrm{cm}] \\
I_{0} & =\text { einfallende Strahlungsleistung } \\
I & =\text { durchgelassene Strahlungsleistung } \\
\tilde{v} & =\text { Wellenzahl }
\end{aligned}
$$

10 Konventionelle, von $\mathrm{H}$. Ew a ld, Ann. Physik (5) 34, 209 [1939], eingeführte Bezeichnung.

11 Jeder Meßpunkt wurde mindestens dreimal gemessen; die Abweichungen der je 3 Messungen untereinander betrugen bei 20000 insgesamt vermessenen $I_{0} / I$-Werten nie mehr als $\pm 3 \%$ ihres Mittelwertes.

12 Der so definierte $f$-Wert bedeutet natürlich nicht mehr als ein konventionelles Maß für die Gesamtabsorption der Bande. 
und die Oszillatorenstärke ${ }^{12}$

$$
\begin{aligned}
& f=A \int k(\tilde{v}) d \tilde{v} \\
A= & 4 \pi \varepsilon_{0} \frac{m c^{2}}{\pi e^{2}} \frac{B a}{2 L} \\
\varepsilon_{0}= & 8,86 \cdot 10^{-14}\left[\mathrm{Amp} \cdot \mathrm{sec} \cdot \text { Volt }^{-1} \mathrm{~cm}^{-1}\right] \\
B= & 10^{-7}\left[\mathrm{Watt}^{-1} \cdot \mathrm{Erg}^{-1}\right] \\
m= & 9,03 \cdot 10^{-28}[\mathrm{~g}] \\
c= & 3 \cdot 10^{10}\left[\mathrm{~cm}^{\left.-\mathrm{sec}^{-1}\right]}\right. \\
e= & 1,60 \cdot 10^{-10}\left[\mathrm{Amp}^{-\mathrm{sec}]}\right. \\
a= & 10^{3}\left[\mathrm{~cm}^{3} \cdot \mathrm{Liter}^{-1}\right] \\
L= & 6,02 \cdot 10^{23}\left[\mathrm{Mol}^{-1}\right] .
\end{aligned}
$$

etwas ungenauer bekannt (s. u.). Doch blieb auch hier der Fehler bei einer. Konzentration unterhalb $1 \%$. Im einzelnen wurde folgendermaßen vorgegangen:

Die Heizspannung an der Lichtquelle (OsramWolfram-Wendellampe $12 \mathrm{~V}, 8 \mathrm{Amp}$.) wurde durch einen röhrengeregelten Umformer auf $\pm 5 \%$ konstant gehalten. Die Wolfram-Wendel wurde in die $\mathrm{Ab}$ sorptionsküvette abgebildet. Hierbei war die wirksame Schichtdicke für die Randstrahlen höchstens $1 \%$ größer als für den Strahl in der Mittelachse. Die Küvette wurde nach Unterdrückung des Streulichtes auf den Spalt eines Zeiß-Dreiprismen-Spektrographen mit Autokollimationskamera $(f=130 \mathrm{~cm})$ abgebildet. Dieser hatte in dem untersuchten Spektralbereich eine Dispersion von etwa $5 \AA / \mathrm{mm}$. Am Spektrographen wurde in der Photoplattenebene ein Spalt angebracht, an dem durch Drehen des Försterlingschen Prismensatzes das Spektrum sauber meßbar vorbeigedreht wurde. Hinter dem Spalt war eine Photozelle angebracht mit einem Kompensations-Röhrenelektrometer ${ }^{13}$. Die bei Belichtung der Photozelle zusätzlich auftretende Gitterspannung steuert den Anodenstrom der einen Röhre; diese Anodenstromänderung wurde über ein Spiegelgalvanometer mittels Fernrohrablesung gemessen. Die Empfindlichkeit, d. h. das Verhältnis Gitterspannungsänderung zu Galvanometerausschlag, betrug in dieser Anordnung 2,6 $10^{-5} \mathrm{~V} / \mathrm{Skt}$. Durch einen Kontrollversuch wurde festgestellt, daß bis zu Spannungsänderungen von $6 \cdot 10^{-2} \mathrm{~V}$ die Ausschläge bis auf Abweichungen von höchstens 1\% linear mit der Spannung gingen.

Als Photozellen ${ }^{14}$ wurden Vakuumphotozellen mit Caesiumantimonschicht verwendet. Es zeigte sich aber, daß von acht bereits ausgesuchten Zellen nur eine

$13 \mathrm{~J} . \mathrm{S}$ ch int $\mathrm{lme}$ is te r, Die Elektronenröhre als physikalisches Meßgerät, S. 61 [1942]; S t e t t n e r S.-B. Akad. Wiss., Wien, Abt. II a, 142, 481 [1933]; F. M ü l l e r u. W. D ü r i h, Physik. Z. 39, 657 [1938].

${ }^{14}$ Zellen von der Fa. Telefunken, Göttingen. Für ihre Ǔberlassung bin ich den HHrn. Dr. Th e il e und Dr. D i e l s zu Dank verpflichtet. die extrem hohen Anforderungen an Empfindlichkeitskonstanz erfüllte. Die sieben anderen zeigten nach Tagen oder Stunden Zu- oder Abnahme der Empfindlichkeit, die durch Belichtung hervorgerufen wurde und bis zu 0,5\% der Anfangsempfindlichkeit pro Min. betrug. Die endlich benutzte Zelle zeigte auch über die größte Meßdauer von 14-18 Stdn. hintereinander keine meßbare Empfindlichkeitsänderung.

Bei den Absorptionsmessungen wurde die Konzentration so stark geändert, daß Schichtdicken von 50 bis $0,2 \mathrm{~mm}$ verwendet werden mußten, um auf alle Fälle optisch dünne Schichten (40\% Durchlässigkeit im Bandenmaximum) zu haben. Dabei wurden oberhalb von $1 \mathrm{~mm}$ Schichtdicke Präzisionsküvetten von Zeiß, unterhalb von $1 \mathrm{~mm}$ folgende Konstruktion benutzt: Zwei dicke Interferometerplatten wurden in einem Aluminiumrohr mittels dicker Gummipolster gegeneinandergepreßt und durch plangeschliffene Schichtdickenringe aus Glas ${ }^{15}$ auf den gew ünschten Abständen gehalten. Die Ringe waren - wie mit einem Zeißschen Dickenmesser nachgemessen wurde bei der kleinsten Schichtdicke bis auf $5 \%$ planparallel. Außerdem wurde der Abstañ der beiden Interferometerplatten, d.h. die Schichtdicke, mit einem Meßmikroskop durch drei in das Aluminiumrohr gebohrte Löcher in zusammengebautem Zustand nachgemessen. Während der Messungen stand die Küvette mit ihren Endflächen stets horizontal; das Lichtbündel ging über zwei Ablenkprismen vertikal hindurch. Um Reflexions- und Streuverluste möglichst zu eliminieren, konnte die mit absorbierender Lösung gefüllte Küvette gegen eine möglichst gleich gebaute, aber mit Wasser gefüllte, durch Schwenken um eine vertikale Achse reproduzierbar ausgetauscht werden. Die dann immer noch verbleibenden verschiedenen Reflexionsvermögen von Salzlösung und Wasser wurden durch schwache Neigung der einen Küvette gegen die Strahlrichtung so weit berücksichtigt, daß der verbleibende Fehler von $I_{0} / I$ sicher nicht größer als $5 \%$ wurde.

Es wurden so bei konstanter Temperatur von $20^{\circ} \mathrm{C}$ $\pm 1^{\circ} \mathrm{C}$ die Absorptionsbande $\mathrm{D}$ bei $5800 \AA$ und die Doppelbande E und F bei $5200 \AA$ folgender Salze in Abhängigkeit von der Konzentration gemessen: Neodymchlorid, Neodymperchlorat, Neodymbromat, Neodymnitrat, Neodymzinknitrat. Von jedem Salz wurden fast gesättigte Lösungen hergestellt, die dann auf dem Weg über Dichtemessungen schrittweise bis 0,01-m. verdünnt wurden. Die Messungen bei noch kleineren Konzentrationen wurden unterlassen, da die Konzentrationsbestimmungen auf diese Weise relativ zu den sonstigen Genauigkeiten zu ungenau geworden wären. Die Relativkonzentrationen in einer Verdünnungsreihe waren bis auf einen maximalen Fehler von 2.10-3 bekannt. Die Absolutkonzentration in jeder Verdünnungsreihe wurde einmal chemisch durch Verglühen des Neodymsalzes zu Neodymoxyd bestimmt ${ }^{16}$.

15 Fa. Winkel-Zeiß, Göttingen.

16 Für die Durchführung der Analysen habe ich den HHrn. Prof. Dr. v. W a r te n b e g u. Dipl.-Chem. Gros s e-R u y k n sehr zu danken. 
Dabei zeigte es sich, daß das Nitrat (nicht das Zinknitrat) und Bromat Spuren von Verunreinigungen enthalten mußten (Farbtönung des Oxyds sehr schwach bräunlich). Diese Verunreinigungen wurden röntgenspektroskopisch in Emission bestimmt ${ }^{17}$. Es ergab sich, daß etwas Praseodym und Samarium vorhanden waren, die zusammen nicht mehr als max. 5\% ausmachten. Dies wurde bei der Bestimmung der Neodymkonzentration berücksichtigt. Die angegebene Absolutkonzentration der Lösungen war also nur mit etwa $\pm 3 \%$ bekannt.

Alle verwendeten Lösungen waren neutral; nur das Nitrat enthielt etwas Säure. die bei der Kristallisation aus schwachsaurer Lösung mit eingeschlossen gewesen war.

Jedesmal nach Durchmessen einer Bande wurde die Messung bei der zuerst gemessenen Wellenlänge wiederholt, um die Konstanz der Meßanordnung während der für eine Bande benötigten Meßzeit von 1 bis 2 Stdn. zu prüfen.

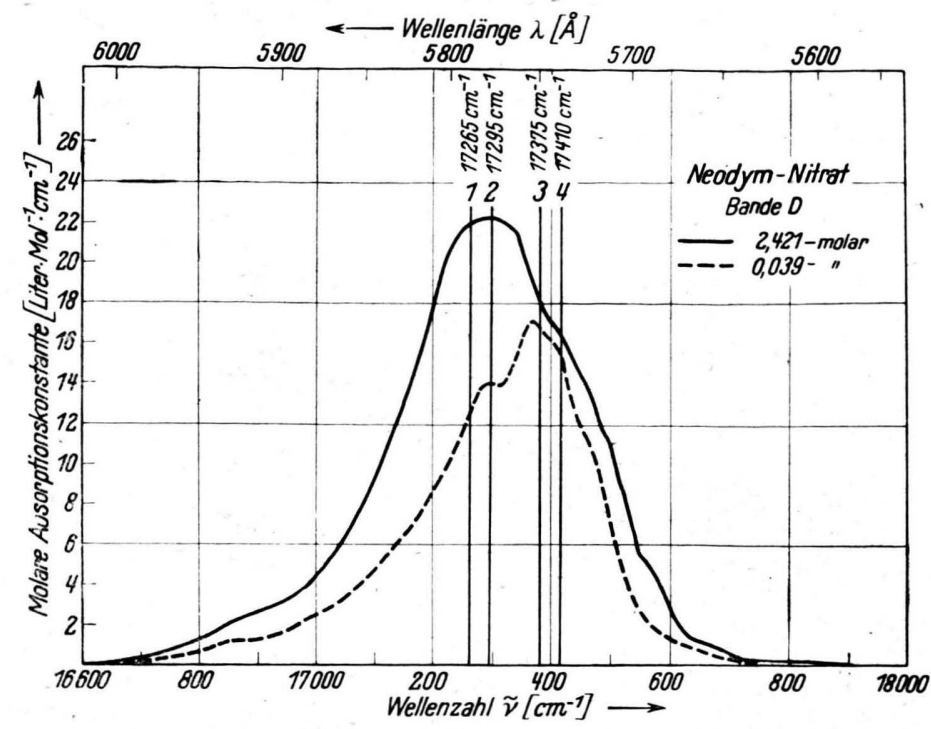

Abb. 1. Molare Absorptionskonstante des Neodymnitrates in der Bande D als Funktion der Wellenzahl bzw. Wellenlänge bei größter und kleinster gemessener Konzentration.

\section{Ergebnisse}

Wir stellen die Meßergebnisse an Hand einiger Abbildungen zusammen. Dabei ist entspr. dem experimentellen Verfahren der Verdünnung um immer denselben Faktor zunächst der logarithmische Maßstab für die Konzentrationen gewählt.

a) Abb. 1 zeigt die Bande $D$ des Neodymnitrates bei der größten

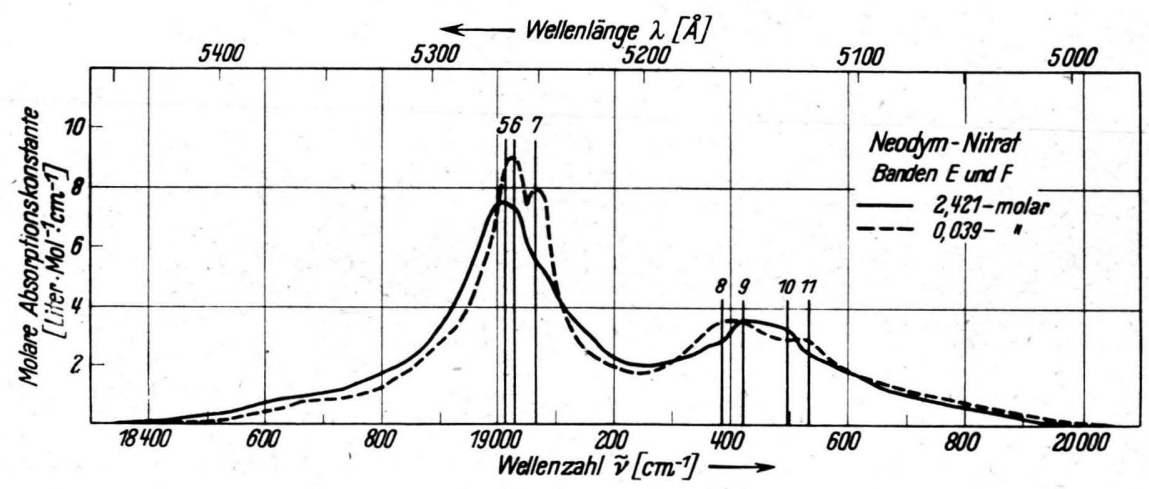

Abb. 2. Molare Absorptionskonstante des Neodymnitrates in den Banden E und $\mathrm{F}$ als Funktion der Wellenlänge bzw. Wellenzahl bei größter und kleinster gemessener Konzentration.

und der um den Faktor 60

verdünnten kleinsten gemessenen Konzentration. Die beiden Kurven sind stark verschieden, und zwar hängt sowohl die Fläche als auch die Form der Bande stark von der Konzentration ab. Die Intensitäten der einzelnen zu der Bande zusammenfließenden Linien hängen also in sehr verschiedener Weise von der Konzentration ab.

Dasselbe gilt auch, wie Abb. 2 zeigt, von der Doppelbande $\mathrm{E}$ und $\mathrm{F}$. In beiden Abbildungen sind aus Gründen der Übersichtlichkeit alle bei Zwischenkonzentrationen aufgenommenen Absorptionskurven fortgelassen. Sie bilden einen

17 Für die Ausführung der Röntgen-Spektralanalyse bin ich den HHrn. Prof. Cor ens u. Prof. Ernst zu Dank verpflichtet. stetigen Ubergang zwischen den beiden gezeichneten Grenzfällen.

b) Allerdings erfolgt dieser Übergang erstens keineswegs monoton in derselben Richtung, und zweitens hängt er stark vom Anion ab. Um das zu zeigen, sind wegen der Unmöglichkeit, das gesamte Beobachtungsmaterial hier zu reproduzieren, die in Abb. 1 und 2 durch vertikale Linien und die Bezifferung 1..11 gekennzeichneten Frequenzen (vielleicht etwas willkürlich) herausgegriffen worden. Die an dieser Stelle gemessenen Absorptionskonstanten - abgekürzt als $k_{1} \ldots k_{11}$ bezeichnet - sind in den Abb. 3 a bis d für die Bande D und den Abb. 4 a bis d für die Bande E, 


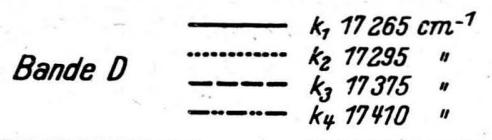

Abb. 3. Konzentrationsabhängigkeit der molaren Absorptionskonstanten bei den in Abb. 1 mit 1, $2,3,4$ bezeichneten Wellenzahlen.

Abb. 4. Konzentrationsabhängigkeit der molaren Absorptionskonstanten bei den in Abb. 2 mit 5, ..., 11 bezeichneten Wellenzahlen.

Abb. 3.
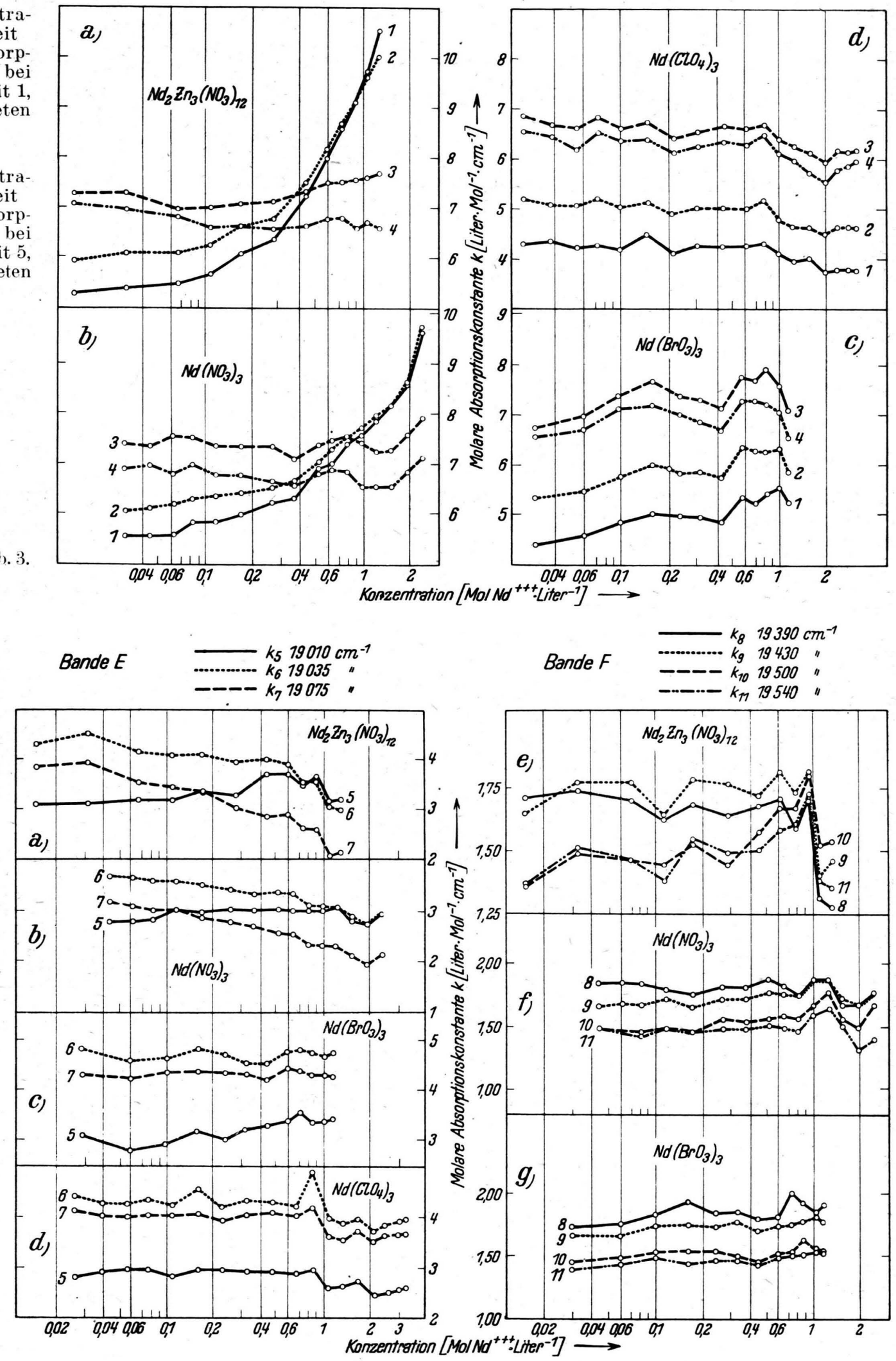

Abb. 4. 
$4 \mathrm{e}$ bis $\mathrm{g}$ für die Bande $\mathrm{F}$ als Funktion der Konzentration aufgetragen.

Es muß darauf hingewiesen werden, daß zwar die charakteristischen Frequenzen für jede Bande ausgesucht wurden, daß ihre Absorptionskonstanten jedoch nicht unbedingt eine eigene physikalische Bedeutung haben müssen, da sich die einzelnen Linien an jeder Stelle der Bande überlagern. Ferner sind die Kurven für das Chlorid nicht mit dargestellt, da sich das Chlorid in jeder

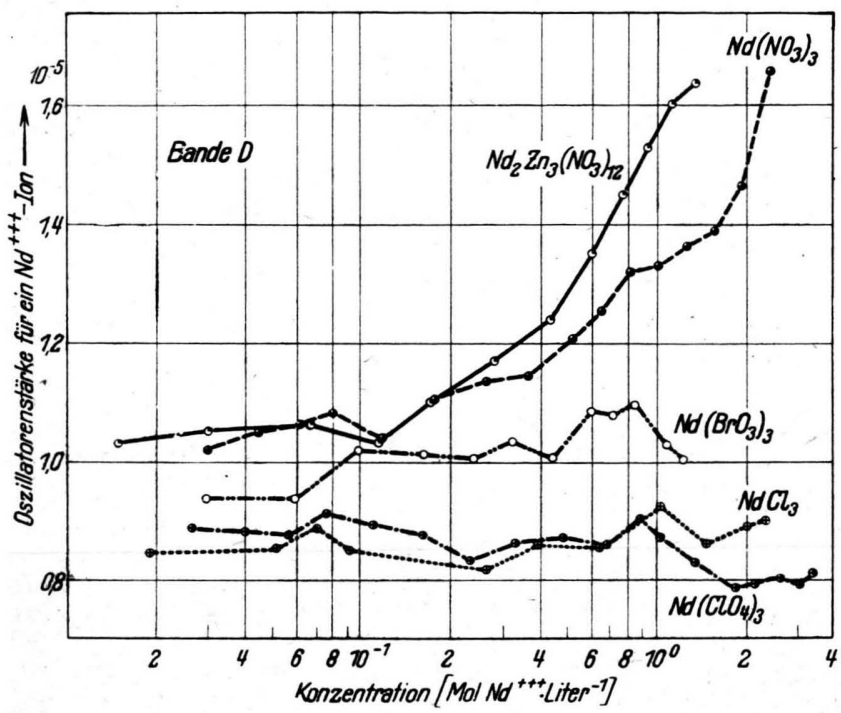

Abb. 5. Oszillatorenstärke je Neodym-Ion der Bande D als Funktion der Konzentration.

Hinsicht wie das Perchlorat verhält. Bei der Bande $\mathrm{F}$ wurde auch auf die Darstellung des Perchlorats verzichtet, da bereits beim Bromat wie beim Chlorid und Perchlorat die Absorptionskonstanten bei den verschiedenen Wellenzahlen praktisch parallel verlaufen.

Alle Abbildungen zeigen übereinstimmend, daß die molare Absorptionskonstante der einzelnen Ionen keineswegs von der Konzentration unabhängig ist. Das Beersche Gesetz gilt also nicht. Es fällt zunächst auf, daß bei allen Frequenzen die Absorptionskonstanten nicht monoton zu- oder abnehmen, sondern mehrfach zwischen maximalen und minimalen Werten hin- und herwandern ${ }^{18}$. Diese Schwankungen, deren Wert bis zu 20\% vom mittleren Wert abweicht, erfolgen erstaunlich steil: Konzentrationsänderungen von manchmal

18 Die Meßfehler sind höchstens von der Größe des Durchmessers der die Meßpunkte umgebenden Kreise.

19 Hier nur in erster Näherung, s. Abschn. d). nur 20\% können von einem Minimum zu einem Maximum führen und umgekehrt. Es scheint, daß dieser „Schwankungseffekt" oberhalb der Konzentration von $0,5-m$. deutlicher ausgeprägt ist als bei kleineren Konzentrationen. Beim Chlorid, Perchlorat und Bromat ${ }^{19}$ hat die Konzentrationsabhängigkeit, wie aus dem praktisch parallelen Verlauf der Kurven in Abb. 3d, $4 \mathrm{~d}$ und $4 \mathrm{~g}$ hervorgeht, für alle Frequenzen denselben Gang: die Kurvenform ändert sich mit der Konzentration praktisch nicht, und zwar ist sie bei allen Konzentrationen identisch mit der Form, wie sie die Banden der beiden Nitrate nur bei der größten Verdünnung haben (gestrichelte Kurven der Abb. 1 und 2). Die Schwankungen sind also Schwankungen der Fläche bei konstanter Kurvenform.

c) Bei den beiden Nitraten überlagert sich jedoch den bei allen Salzen auftretenden, eben beschriebenen Schwankungen eine sehr auffällige Erscheinung. Wir betrachten zunächst die BandeD. Wie die Abb. $3 \mathrm{a}$ und $3 \mathrm{~b}$ zeigen, steigt die $\mathrm{Ab}$ sorptionskonstante für die Frequenzen 1 und 2 steil mit der Konzentration an, während die Absorptionskonstante bei den Frequenzen 3 und 4 nur die oben beschriebenen Schwankungen erleiden. Dadurch wird die in Abb. 1 gezeigte Deformation der langwelligen Seite der Bande ${ }^{20}$ und gleichzeitig èine Vergrößerung der Fläche bewirkt, auf die wir noch zurückkommen. Die langwellige Seite der Bande ist also gegen Anderung der Konzentration besonders ,empfindlich“, und zwar ist die „Empfindlichkeit“ beim Doppelnitrat noch größer als beim einfachen Nitrat.

d) Beim Perchlorat und Chlorid ist von dem geschilderten, in der verschiedenen „Empfindlich. keit" verschiedener Frequenzen ein und derselben Bande bestehenden „Nitrat-Effekt", wie oben gesagt, nichts zu merken. Beim Bromat jedoch ist er schwach angedeutet: Nach Abb. $3 \mathrm{c}$ und $4 \mathrm{c}$ sind auch hier die Frequenzen 1, 2 und 5 in derselben Weise ausgezeichnet wie bei den Nitraten, nur in wesentlich geringerem Maße.

Wir haben also in bezug auf den „NitratEffekt" ein Empfindlichkeitsgefälle vom Doppelnitrat über Nitrat und Bromat zum Perchlorat und Chlorid, wobei die beiden Nitrate auf der

\footnotetext{
20 Für das Doppelnitrat sind die Kurven fast dieselben wie die hier reproduzierten des Nitrates. Sie sind, mit der geringeren Genauigkeit der photographischen Methode gemessen, bei $\mathrm{Chow}_{\mathrm{w}} \mathrm{Hell}$ w e g e ${ }^{9}$ (Abb.2) ausführlich dargestellt.
} 


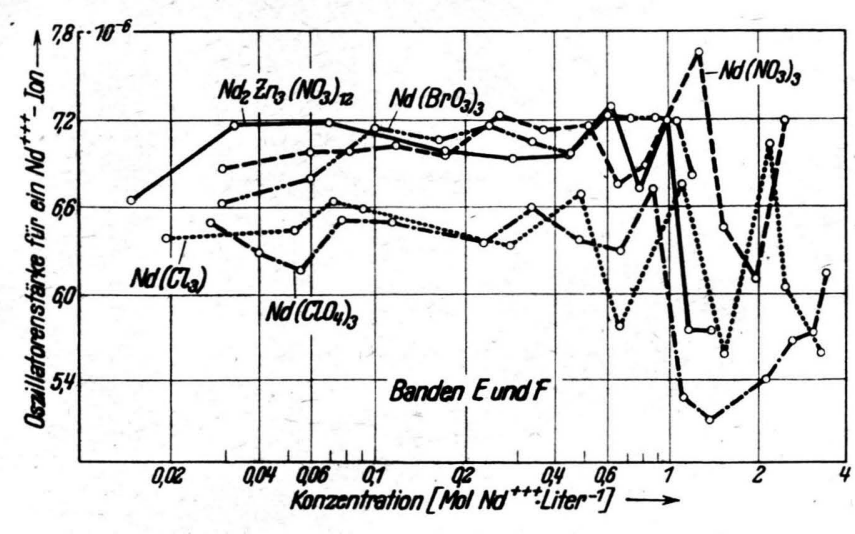

Abb.6. Gesamtoszillatorenstärke je Neodym-Ion der Banden $\mathrm{E}$ und $\mathrm{F}$ als Funktion der Konzentration. tuell von kleinen Verschiebungen abgesehen, bei denselben Konzentrationen aufzutreten, was selbst noch an den steilen Nitratkurven erkennbar ist ${ }^{21}$. Bei den Banden $\mathrm{E}$ und $\mathrm{F}$ sind die Verhältnisse komplizierter, da sich nur die Gesamtfläche, d.h. die Summe der beiden Oszillatorenstärken, messen läßt.' Außerdem hat (vgl. Abb. 2) die Absorption an verschiedenen Stellen der Banden einen entgegengesetzten Gang mit der Konzentration, so daß bei der Integration ein gewisser Teil der Konzentrationsabhängigkeit verloren geht.

Es ist also bei der Doppelbande der gesamte $f$ Wert physikalisch nicht so bedeutungsvoll wie bei der Bande D. Trotzdem zeigt die Abb. 6 deutlich noch einmal folgende Tatsachen: 1 . Der $f$-Wert ist einen und das Perchlorat und Chlorid auf der anderen Seite sich untereinander sehr ähnlich verhalten, während das Bromat eine klare Mittelstellung einnimmt.

e) Diese Mittelstellung ist besonders deutlich zu sehen an der Fläche, d.h. der Oszillatorenstärke der Bande. In Abb. 5 ist die Oszillatorenstärke der Bande D als Funktion der Konzentration dargestellt.

Bei den Nitraten fällt sofort der (beim Doppelnitrat besonders) steile Anstieg oberhalb des schon von Chow und Hellwe $\mathrm{e} \mathrm{e}^{9}$ mit der photographischen Methode gefundenen „Knicks“ bei etwa 0,1-m. auf. Das Perchlorat und Chlorid zeigen diesen Effekt nicht; abgesehen von dem „Schwankungseffekt" bleibt der $f$-Wert ziemlich konstant. Beim Bromat jedoch ist deutlich ein den Schwankungen überlagerter Anstieg mit wachsender Konzentration vorhanden, der jedoch viel schwächer ist als bei den Nitraten. Außerdem liegt die Bromatkurve auch den Absolutwerten nach zwischen denen des Chlorids und Perchlorats auf der einen und denen der beiden Nitrate auf der anderen Seite. Die bei allen Salzen auftretenden "Schwankungen“ scheinen im übrigen, even-

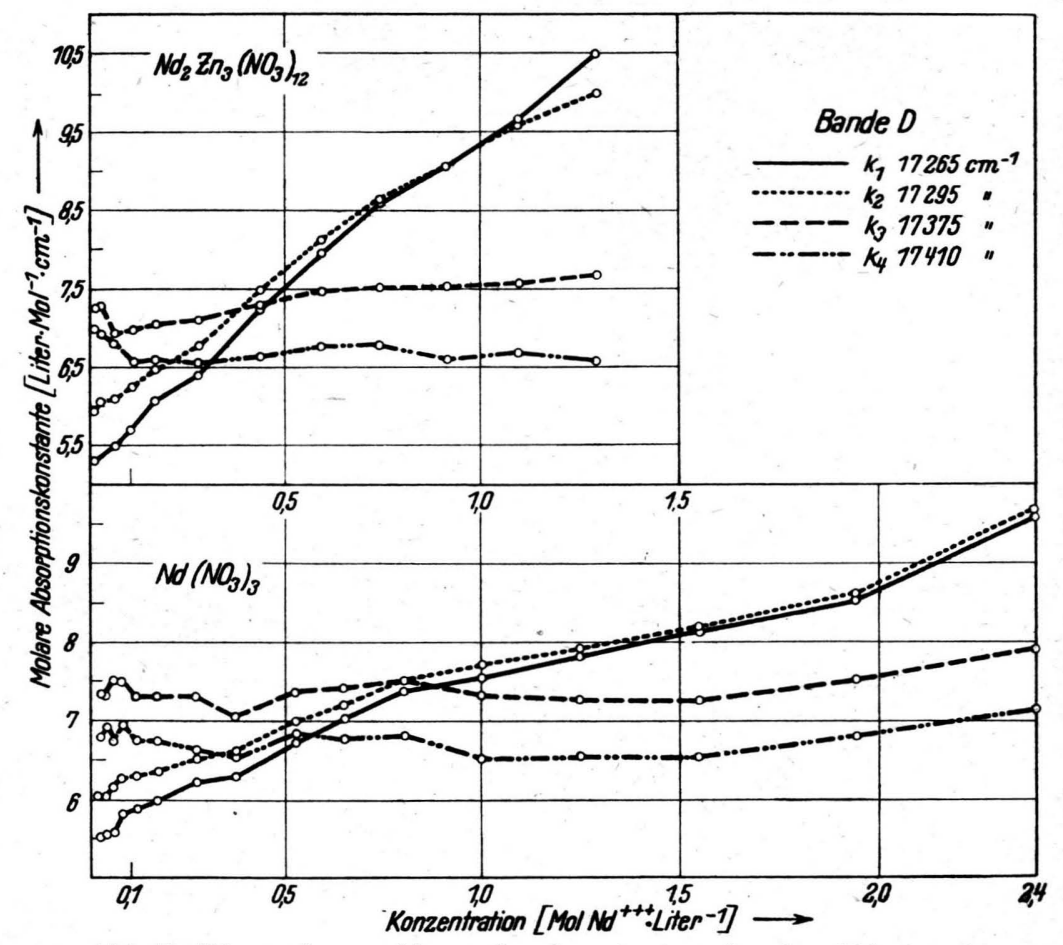

Abb. 7. Die molaren Absorptionskonstanten der in Abb. 1 gekennzeichneten Wellenzahlen in der Bande $D$ von Neodymzinknitrat und Neodymnitrat als Funktion der linear aufgetragenen Konzentration. beim Chlorid und Perchlorat kleiner als bei den Nitraten und dem Bromat; er liegt bei der kleinsten Konzentration zwischen diesen Grenzfällen.

2. Wie schon bei der Bande D betont, sind auch hier die Schwankungen oberhalb von $0,5-m$. besonders deutlich.

21 Diese Tatsache läßt sich natürlich auch aus den Abb. $3 \mathrm{a}$ und $4 \mathrm{a}$ ablesen. 
3. Die Schwankungen erfolgen bei allen Salzen bei annähernd denselben Konzentrationen, wenn man den relativ großen Abstand der Meßpunkte în Rechnung setzt.

4. Eine monotone Änderung der Oszillatorenstärke oberhalb von 0,1-m., wie bei der Bande D infolge eines „Nitrat-Effektes“, ist aus oben erwähnten Gründen der oft gegenläufigen Änderungen der einzelnen Absorptionskonstanten mit der Konzentration nicht von den Schwankungen zu trennen.

Benutzt man für die Konzentration statt des logarithmischen den linearen Maßstab, so ergibt sich, wie die Abb. 7 zeigt, der steile Anstieg der Absorptionskonstanten infolge des ,Nitrat-Effektes" als streckenweise lineare Funktion der Konzentration.

Durch die Knicke in diesen und den vorhergehenden Kurven sind ganz bestimmte Konzentrationen ausgezeichnet. Berücksichtigt man, daß diese Konzentrationen sich nur mit einer Ungenauigkeit festlegen lassen, die etwa durch den Abstand zweier Meßpunkte gegeben ist, so ergeben sich aus allen gemessenen Kurven und für alle Salze dieselben fünf ausgezeichneten Konzentrationen: etwa 0,1-m., 0,4-m., 0,8-m., 1,1-m. und 2,0-m.

\section{D e utung}

Wir behandeln zunächst den „Nitrat-Effekt“. Nach den oben angegebenen Arbeiten von F r e e d und Mitarbb. ${ }^{7}$ besteht dieser Effekt in Lösungen von Europiumnitrat darin, daß zunächst bei genügend kleiner Konzentration alle EuropiumIonen dieselbe, durch eine bestimmte Symmetrie ausgezeichnete Umgebung besitzen, daß aber bei wachsender Konzentration die Umgebung einer wachsenden Zahl der Ionen spontan in eine andere Struktur mit einer anderen Symmetrie umklappt, bis schließlich bei der gesättigten Lösung die Umgebung praktisch aller Ionen die neue Struktur besitzt. Die Symmetrie dieser ,Sättigungsstruktur" ist wahrscheinlich höher als die der ,Verdünnungsstruktur“, da sie sich im Spektrum durch ein Aufspaltungsbild von nur 3 Linien

\footnotetext{
22 Je höher die Symmetrie des Kristallfeldes ist, desto kleiner wird die Zahl der Komponenten, in die die einzelnen Terme des Ions aufspalten. Da auch die Zahl der Übergangsverbote mit wachsender Symmetrie zunimmt, muß die Zahl der Linien abnehmen (s. K. H. H e 11 w e ge, Nachr. Ges. Wiss. Göttingen, Math.physik. Kl. 1947, 37.
}

gegenüber 4 Linien bei starker Verdünnung auszeichnet ${ }^{22}$.

Wegen des praktisch gleichen Wertes der Ionenradien beim Europium ${ }^{+++}(1,15 \AA)$ und Neodym $+++(1,3 \AA)$ bestehen wohl keine Bedenken, das Freedsche Ergebnis auf das Neodymnitrat und, da sich bis auf quantitative Differenzen das Neodymzinknitrat ebenso verhält, auch auf dieses zu übertragen. Wir müssen also annehmen, daß außer bei den kleinsten und vielleicht auch bei den größten Konzentrationen jede Absorptionsbande eine Überlagerung von zwei ${ }^{23}$ Spektren (zwei Aufspaltungsbildern) darstellt, deren Intensität sich mit der Konzentration verschiebt.

Allerdings lassen sich, im Gegensatz zum Europium, die Aufspaltungsbilder beim Neodym aus den schon oben angeführten Gründen nicht trennen. Aus der Tatsache, daß mit steigender Konzentration die Absorption pro Ion an der langwelligen Seite der Bande D stark ansteigt, folgt jedoch erstens, daß hier die stärkeren Linien des zu der „Sättigungsstruktur“ gehörenden Aufspaltungsbildes liegen, und zweitens, daß die bei der ,Sättigungsstruktur“ vorhandene gesamte Oszillatorenstärke der Bande D wesentlich stärker ist als die bei der „Verdünnungsstruktur" vorhandene. Nimmt man an, daß bei gesättigter Lösung das Umklappen der Struktur wirklich wie nach F r e ed beim Europiumnitrat bei allen Ionen bereits erfolgt ist, so ist das Verhältnis der Oszillatorenstärke mindestens gleich 1,7 , wie aus Abb. $5 \mathrm{zu}$ ersehen ist ${ }^{54}$. Möglicherweise jedoch ist dieser Wert noch zu klein, da die Kurve der Oszillatorenstärke in der Nähe der Sättigung noch weiter ansteigt, d. h. die Strukturumwandlung noch nicht vollständig vollzogen ist ${ }^{25}$.

Der bei allen fünf untersuchten Salzen vorhandene "Schwankungseffekt" ist beim augenblicklichen Stand der Untersuchungen noch nicht so eindeutig zu verstehen wie der "Nitrat-Effekt“. Nach der Theorie der erzwungenen elektrischen

${ }^{23}$ Da F r e e d das Europiumzinknitrat nicht untersucht hat, ist beim Neodymzinknitrat diese Zahl nicht gesichert. Möglicherweise kommen mehr als zwei Formen vor.

${ }^{24}$ Y. K. Ch ow u. K. H. H e $1 \mathrm{l}$ w e g e ${ }^{9}$, die bis $\mathrm{zu}$ kleineren Konzentrationen gemessen haben, finden einen Wert von mindestens 2.

25 Es könnte allerdings dieser Anstieg auch dadurch erklärt werden, daß bei steigender Konzentration infolge Abnahme der Ionenabstände die Störfeldstärke wächst, ohne daß die Symmetrie sich ändert. 
Dipolstrahlung müssen die Schwankungen der Absorption ebenfalls auf Strukturänderungen in der Umgebung aller oder eines Teils der SeltenenErd-Ionen zurückgeführt werden, und zwar, da sie bei allen Salzen bei etwa denselben schon oben angeführten Konzentrationen auftreten, auf Struk turänderungen, die von der beim "Nitrat-Effekt" auftretenden Strukturumwandlung und praktisch auch vom Anion unabhängig sind. Die Ansicht, daß es sich wirklich um Strukturänderungen han-

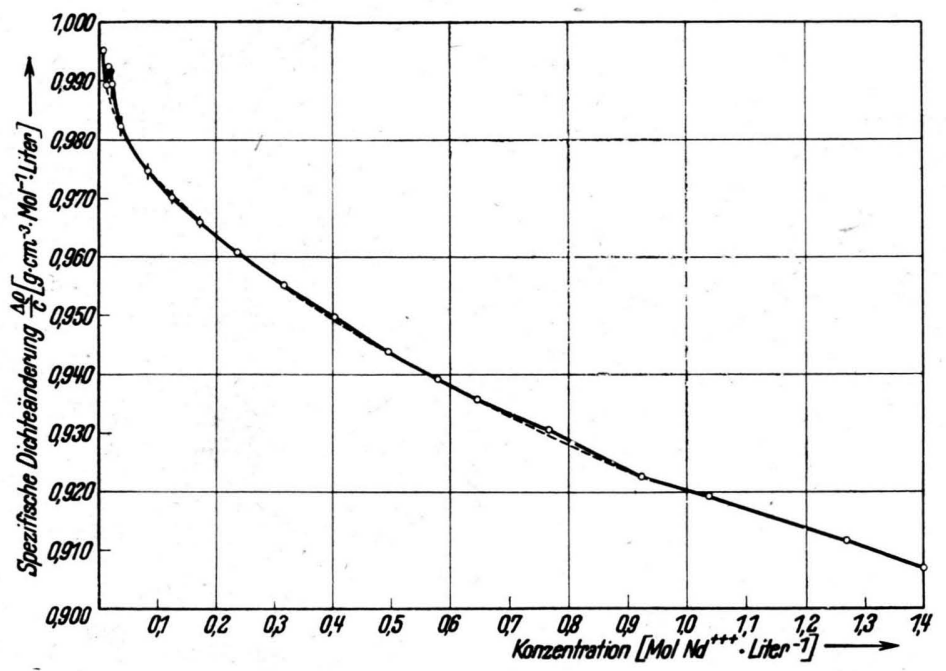

Abb. 8. Die spezifische Dichteänderung einer Neodymzinknitratlösung als Funktion der Konzentration. Die große Meßgenauigkeit (maximaler Fehler gleich halbem Durchmesser der Kreis'e bzw. halber Länge der Vertikalstriche) kommt in der Abb. nicht zum Ausdruck.

delt, wird zusätzlich dadurch gestützt, daß sie von Dichteänderungen begleitet $\mathrm{zu}$ sein scheinen.

In der Abb. 8 ist das Ergebnis einer früher durchgeführten Dichtemessung ${ }^{26}$ am Neodymzinknitrat dargestellt, und zwar ist die spezifische Dichteänderung

$$
\frac{\Delta Q}{c}=\frac{\varrho_{\text {Lösung }}-\varrho_{\mathrm{H}_{2} \mathrm{O}}}{c}
$$

als Funktion der Konzentration $c$ aufgetragen.

26 G. W. O e t j e n, Göttinger Diplom-Arbeit 1945.

27 Die maximalen Fehler sind im rechten Teil der Kurve gleich dem halben Durchmesser des den Meßpunkt umgebenden Kreises, im linken Teil gleich der halben Länge des vertikalen Striches, unterhalb von 0,05-m. überhaupt nicht mehr angegeben. Wegen des kleinen Formates und der Strichdicke der Abb. 8 kommt die erreichte Meßgenauigkeit nicht richtig zum Ausdruck.
Bei den bereits optisch ausgezeichneten Konzentrationen 0,4- und 0,8- $\mathrm{m}$. fallen die Meßpunkte deutlich aus der Kurve heraus, während bei etwa 0,9 - bis $1,0-m$. die Kurve knickartig einen flacheren Verlauf als bei kleineren Konzentrationen bekommt.

Da der maximale Fehler ${ }^{27}$ nach kleineren Konzentrationen hin zunimmt, ist die Konzentration bei $0,1-m$. nicht ganz sicher ausgezeichnet. Wenn man jedoch zugibt, daß sich beim Ergebnis der Dichtemessungen die verschiedenen möglichen Fehler der Teilmessungen nicht gerade addieren werden, die wahren Fehler also kleiner sind als die angegebenen maximalen, so würden sich selbst noch die optisch gefundenen Strukturänderungen unterhalb von $0,05-m$. in der Dichtemessung wiederfinden ${ }^{28}$.

Auf Grund der geschilderten experimentellen Ergebnisse wird man sich die Vorgänge bei wachsender Konzentration vielleicht folgendermaßen vorstellen können:

Da alle untersuchten Salze bei genügend starker Verdünnung nach Form und Fläche dieselben Absorptionsbanden haben, muß hier die Umgebung der Neodym-Ionen, soweit sie zum optisch-wirksamen Störfeld beiträgt, unabhängig vom Anion immer dieselbe sein. Die Struktur in der nächsten Umgebung des Ions wird von der Einstellung der Wasserdipole im Ionenfeld bestimmt (Hydrathülle) und geht über mehrere Schichten von Wassermolekülen hinweg nach außen in die durch Ionen nicht mehr gestörte Struktur des ,normalen“ Wassers über. Mit wachsender Konzentration wird dieses ,normale" Wasser bis zum vollständigen Verschwinden immer weniger, der Abstand zwischen den Ionen kleiner, und die Hydrat-

28 Diese Parallelität zwischen der optischen und der Dichtemessung erscheint um so eindringlicher, da die Dichtemessung $\mathrm{zu}$ einer Zeit gemacht wurde, als der Schwankungs-Effekt noch völlig unbekannt war. Die beiden Methoden sind also auch psychologisch unabhängig gewesen. Da die Konzentrationsänderungen durch schrittweises Verdünnen erfolgten, ein Meßfehler sich also fortgepflanzt hätte, ist das Herausfallen einzelner Punkte aus der Kurve wohl kein Meßfehler. 
hüllen werden von außen her um- und abgebaut ${ }^{29}$.

Nach dem Freedschen Ergebnis und den vorliegenden Messungen muß dieser angenommene Umbau der Hydrathüllen beim Chlorid bis zur Sättigung, wo schließlich nur noch wenige Wassermoleküle auf ein Seltenes-Erd-Ion kommen, ohne Anderung der Symmetrie des Störfeldes erfolgen. Aber auch die Stärke des Störfeldes ändert sich nach unseren Messungen nur so wenig, daß die Oszillatorenstärke um nicht mehr als 10-20\% schwankt. Man muß also wohl annehmen, daß die nächste Umgebung der Neodym-Ionen im Chlorid (und ebenso im Perchlorat) bis hinauf zur Sättigung praktisch unverändert bleibt und daß die Schwankungen der Oszillatorenstärke mit wachsender Konzentration ihre Ursache in Strukturänderungen haben, die sich auf die weitere Umgebung des Neodym-Ions beschränken und sich in der $4 f$-Schale nur wenig bemerkbar machen. Bei den Nitraten dagegen gibt es neben diesen Strukturänderungen in der Umgebung einer mit der Konzentration wachsenden $Z$ ahl von Ionen die nach dem experimentellen Befund zunächșt mit „Nitrat-Effekt" bezeichnete radikale Strukturänderung, bei der nicht nur die Stärke, sondern nach Freed auch die Symmetrie des Störfeldes eine ganz andere wird. Hierbei wird also sicher auch der Aufbau der nächsten Umgebung des NeodymIons geändert. Mehr chemisch kann man von

29 Möglicherweise läßt sich das Konzentrationsgebiet, in dem das letzte ,normale" Wasser herausgezogen wird, mit $0,5-$ bis $0,9-m$. angeben, da von diesem Bereich an der „Schwankungseffekt" merklich stärker wird. Ferner erhält gerade in diesem Bereich die $\Delta \mathrm{\varrho} / c$-Kurve eine andere Neigung (s. Abb. 8). Auch die Zahl von $10-12$ Wassermolekülen, die bei etwa 0,7-m. im Mittel auf 1 Ion kommen, erscheint durchaus sinnvoll für eine solche Hydrathülle. einer Komplex- oder Molekülbildung sprechen. sofern man bedenkt, daß es sich dabei nicht um den Grenzfall handelt, bei dem sich eine gemeinsame Elektronenhülle bildet (Bindung durch Austauschkräfte), sondern mehr um den entgegengesetzten Grenzfall der Wechselwirkung zwischen den Ionen untereinander einerseits und den Ionen und den Wasserdipolen andererseits (elektrostatische Bindung).

Das Bromat nimmt eine Mittelstellung zwischen den Nitraten auf der einen und dem Chlorid und Perchlorat auf der anderen Seite ein. Hier ist also wahrscheinlich der an der Komplexbildung beteiligte Prozentsatz der Neodym-Ionen oder die beim Umbau eintretende Änderung des Störfeldes kleiner.

Die Frage, warum alle Salze den ,Schwankungs-Effekt", nicht aber alle den „Nitrat-Effekt" zeigen, läßt sich vielleicht wie folgt beantworten: Das Cl- und das $\mathrm{ClO}_{4}$-Ion haben praktisch Kugelgestalt. Dagegen ist das $\mathrm{NO}_{3}$-Ion ein ebenes Dreieck, während das $\mathrm{BrO}_{3}$-Ion eine Pyramide darstellt. Dieser sterische Unterschied muß entscheidend für den „Nitrat-Effekt“ sein, dagegen kann der bei allen Salzen praktisch gleiche „Schwankungseffekt" in erster Näherung nur durch die Wirkung einer allen Anionen gemeinsamen Eigenschaft erklärt werden, nämlich durch die einfach negative Ladung, unabhängig vom sterischen Bau des Anions.

Die Arbeit wurde im II. Physikalischen Institut der Universität Göttingen ausgeführt. Ich danke Hrn. Doz. Dr. H e $1 \mathrm{l}$ w e g e für Anregung und Förderung, ferner Hrn. Prof. v. W a r te n b e r g u. Hrn. Dipl.Chem. Grosse-R u y cken sowie den HHrn. Dr. Di e ls, Dr. Pa ul u. Dr. Theile für experimentelle und apparative Hilfe. 